\title{
Detección e intervención en personas de riesgo de padecer diabetes en farmacias comunitarias de la provincia de Valencia (DETECTA SUCRE)
}

\author{
Óscar García Agudo ${ }^{1}$, Esther Desirée Ruiz González², Vicente Colomer Molina ${ }^{2,3}$, María Gil Peinado ${ }^{10}$, \\ José Sendra-Lillo $2,3,40$
}

1. Farmacéutico del Centro de Información del Medicamento y Atención Farmacéutica del Muy llustre Colegio Oficial de Farmacéuticos (MICOF) de Valencia. 2. Farmacéutico comunitario. 3. Vocal del Muy Ilustre Colegio Oficial de Farmacéuticos de Valencia. 4. Doctor en Farmacia.

\section{PALABRAS CLAVE}

Diabetes mellitus tipo 2, cribado, riesgo, farmacia comunitaria

\section{KEYWORDS}

Type 2 diabetes mellitus, screening, risk, community pharmacy

\section{RESUMEN}

Objetivo: detectar personas en riesgo alto o muy alto de padecer diabetes mellitus tipo 2 (DM2) o alteraciones del metabolismo de los hidratos de carbono, derivación para posible diagnóstico al médico y realizar una intervención educativa.

Métodos: estudio observacional transversal y longitudinal prospectivo con intervención educativa en farmacias comunitarias situadas en la provincia de Valencia entre junio y noviembre de 2018. Se incluyeron usuarios de las farmacias comunitarias, mayores de 18 años, no diagnosticados de DM2 que aceptaron realizar la encuesta. Muestreo no probabilístico.

Variable principal: puntuación en el test Findrisc, determinación capilar (si $F \geq 15$ ), intervención y número de diagnósticos de DM2 y preDM2. Las diferencias entre grupos se calcularon con el test de chi-cuadrado, t de Student o de Wilcoxon.

Resultados: el estudio se realizó en 25 farmacias. La muestra incluyó a 752 usuarios. De ellos, 148 $(19,7 \%)$ tenían alto o muy alto riesgo de DM2 $(F \geq 15)$. Se realizaron 118 determinaciones capilares válidas (22 no aceptaron y 8 no la repitieron), 78 (10,4\%) tuvieron resultados superiores a los normales y a 48 (6,4\%) se les derivó al médico. 32 de estos no fueron al médico o no informaron al farmacéutico del resultado. De los 16 que conocemos el resultado, 12 (1,6\%) fueron diagnosticados de diabetes o prediabetes.

Conclusiones: los resultados de este estudio piloto dejan entrever la utilidad de la farmacia comunitaria como agente con un papel importante en el cribado de DM2 al detectar el presente trabajo casi un $20 \%$ de usuarios con un riesgo elevado de padecer la enfermedad.

\section{Detection and intervention of people at risk of diabetes in community} pharmacies of Valencia

\section{ABSTRACT}

Objective: To detect people at high or very high risk of diabetes (DM2) or alterations in carbohydrates metabolism, referral for possible medical diagnosis and to implement educational intervention.

Methods: A cross-sectional and longitudinal prospective observational study was carried out in 25 community pharmacies in Valencia between June-November 2018. Pharmacy users over 18 years old, not diagnosed with DM2 and who agreed to participate in the survey were included in the study. Nonprobabilistic sampling was made.

Primary endpoint: Findrisc questionnaire score, capillary blood measurement (if $\mathrm{F} \geq 15$ ), intervention and number of DM2 and preDM2 diagnosed. Differences between the groups were calculated using Chisquared test, Student's t test, or Wilcoxon test.

Results: This study was carried out in 25 pharmacies. The sample included 752 users. Of them, 148 $(19,7 \%)$ with a high or very high risk of DM2 ( $\mathrm{F} \geq 15) .118$ valid capillary determinations were performed (22 did not accept and 8 did not repeat it), 78 (10.4\%) had higher than normal results and 48 (6.4\%) were referred to the doctor. 32 of these did not go to the physician or did not inform the pharmacist of the result. Of the 16 that we know the result, 12 (1.6\%) were diagnosed with diabetes or preDM2.

Conclusions: The results of this pilot study suggest the usefulness of the community pharmacy as an agent with an important role in the screening of DM2, as this study detected almost 20\% of users at high risk of DM2.

Un resumen de este trabajo se presentó como comunicación en formato póster en el 1er Congreso Nacional SEMERGEN-SEFAC en 2019.

Recibido: 15/04/2021

Aceptado: 13/07/2021

Disponible online: 11/01/2022
Financiación: el programa fue financiado por el MICOF integramente, a excepción de la participación de los laboratorios
Abbott, Lifescan, Ascensia, Menarini y Novalab, que proporcionaron los dispositivos necesarios para este pilotaje.
Conflicto de intereses: ninguno.
Cite este artículo como: Garcia Ó, Ruiz ED, Colomer V, Gil M, Sendra-Lillo J. Detección e intervención en personas de
riesgo de padecer diabetes en farmacias comunitarias de la provincia de Valencia (DETECTA SUCRE). Farmacéuticos Co-
munitarios. 2022 Jan 11; 14 (1): 16-26. doi:10.33620/FC.2173-9218.(2022/Vol14).001.4
Correspondencia: Óscar Garcia Agudo (oscar.garcia@micof.es).
ISSN 1885-8619 @SEFAC (Sociedad Española de Farmacia Clínica, Familiar y Comunitaria). Todos los derechos reservados. 


\section{INTRODUCCIÓN}

La Encuesta Nacional de Salud en 2017 estimó la prevalencia de DM2 en España en el 7,8\% (7,1\% en mujeres y $8,5 \%$ en hombres) (1). Un estudio en España identificó que casi el $30 \%$ de la población de estudio tenía algún tipo de alteración del metabolismo de los hidratos de carbono y que la prevalencia global de DM2 ajustada por edad y sexo era del $13,8 \%$, de los cuales aproximadamente la mitad (6\%) tenía DM2 no conocida (2), cifras similares a las obtenidas previamente en el Estudio Valencia (3) (14,8\%), realizado por la Generalitat Valenciana en la provincia de Valencia.

Las personas con DM2 no diagnosticada presentan alto riesgo de padecer enfermedades cardiacas, dislipidemia, hipertensión y obesidad en comparación con la población no diabética; por ello, la detección precoz y el tratamiento inmediato reducen la progresión y gravedad de la enfermedad, así como las futuras complicaciones e ingresos hospitalarios $(4,5)$.

El término 'prediabetes' incluye la presencia de una glucemia basal alterada (GBA), de una intolerancia a la glucosa (ITG) o de ambas condiciones a la vez (GBA+ITG), situaciones todas ellas que implican un riesgo elevado de desarrollar DM2 y de sufrir complicaciones cardiovasculares (6).

Existen varias estrategias para el cribado de DM2, siendo el test Findrisc (FINish Diabetes RIsc SCore) una de las herramientas más eficientes de cribado (7), recomendado por instituciones sanitarias relevantes como el The National Institute for Health and Care Excellence (NICE) (8), Canadian Task Force (9) y la Sociedad Española de Diabetes (SED)(10). Se ha utilizado en campañas públicas (11) de detección de la DM2, así como en el cribado en farmacias comunitarias (12-16).

Dada la accesibilidad y proximidad a la población de las farmacias comunitarias, el Muy Ilustre Colegio Oficial de Farmacéuticos de Valencia (MICOF) diseñó el programa piloto DETECTA SUCRE cuyo objetivo fue pilotar la viabilidad de que los farmacéuticos comunitarios detectasen personas en riesgo de padecer alteraciones del metabolismo de los hidratos de carbono, modificar dicho riesgo con la intervención del farmacéutico y su posterior derivación para el posible diagnóstico en los centros de salud de atención primaria.

Además, trabajó con distintos objetivos específicos (17):

- Detectar personas con riesgo alto y muy alto de padecer DM2 utilizando el test de Findrisc.

- Confirmar la situación anterior en relación con el valor capilar de la glucemia basal, azar o hemoglobina glicada.

- Caracterizar y realizar una intervención (hábitos saludables, factores de riesgo de desarrollar la enfermedad, educación sanitaria, etc.) en los pacientes en función del riesgo de padecer DM2.

- Derivar al médico de atención primaria (MAP) a aquellos usuarios con sospecha de DM2 o preDM2 para posible diagnóstico.

\section{MATERIAL Y MÉTODOS}

Estudio observacional descriptivo transversal. Adicionalmente se realizó trabajo de tipo longitudinal prospectivo en el caso de los individuos que recibieron una intervención educativa en farmacias comunitarias.

El estudio se llevó a cabo en farmacias situadas en la provincia de Valencia en las cuales hubiera al menos un farmacéutico comunitario capacitado previamente en el programa de Cribado de Diabetes DETECTA SUCRE creado por el MICOF. El programa de cribado se llevó a cabo entre el 1 de junio y el 30 de noviembre de 2018.

\section{Criterios de inclusión}

Usuarios de la farmacia comunitaria, mayores de 18 años, no diagnosticados de DM2 y que aceptaron realizar el test tras el ofrecimiento por parte del farmacéutico comunitario.

\section{Criterios de exclusión}

Se excluyó a mayores de 18 años no capacitados para cumplimentar el test o personas que no aceptaron el ofrecimiento. Usuarios diagnosticados de DM2 mellitus y/o que tomaran medicamentos para la DM2.

\section{Variables y sistemas de medición}

Las variables de estudio son aquellas recogidas en el test de Findrisc: edad (años), sexo (hombre/mujer) y puntuación total del test de Findrisc (puntos). Se establecieron 5 subgrupos de riesgo: bajo: <7 puntos, ligeramente elevado: 7-11 puntos, moderado: $12-14$ puntos, alto: $15-20$ puntos y muy alto: $>20$ puntos.

Otras variables recogidas fueron:

Número de diagnósticos de DM2 y prediabetes tras la derivación, recogida mediante la información del paciente, del médico o en base al tratamiento pautado. Glucemia basal capilar (GBC) (mg/dL), glucemia al azar capilar (GAC) (mg/dL), ambas recogidas mediante los glucómetros validados de las marcas Accu-chek Aviva, Bgstar, Contour Next, Freestyle Optium, Glucocard G Sensor, Glucomen Areo y LX, Onetouch Select Plus, Onetouch Ultra y Ultra Plus.

Hemoglobina glicada (HbA1c) (\%), determinada con el sistema Cobas b 101 de Roche Diagnostics. Peso y talla, determinados con báscula electrónica calibrada con tallímetro o báscula electrónica calibrada y tallímetro manual validado. Índice de masa corporal $(\mathrm{IMC})\left(\mathrm{kg} / \mathrm{m}^{2}\right)$. Perímetro de cintura, medido con una cinta métrica inextensible. Realización de actividad física (si/no), en función de si realizaba más de 30 minutos al día. Frecuencia diaria de consumo de frutas y verduras (sí/no). Utilización de medicamentos para la presión arterial (si/no), en base a si el paciente cuenta con un fármaco cuya indicación es "hipertensión arterial", valores anteriores de glucosa altos (si/no), familiares con DM2 (no $/ 1^{\text {er }}$ grado $/ 2^{\circ}$ grado). Todos estos criterios se basaron en la clasificación realizada en el test de Findrisc.

Al tratarse de un estudio piloto no se determinó el tamaño muestral. 


\section{Procedimiento}

Los farmacéuticos participantes recibieron una formación semipresencial específica sobre el servicio de cribado de DM2 y prediabetes. El procedimiento seguido fue el siguiente:

1. Captación activa y registro de los datos del participante, realizando las medidas antropométricas correspondientes (peso, talla y perímetro de cintura), incluidas en el test de Findrisc, determinando el grado de riesgo de padecer DM.

2. Si la puntuación del cuestionario de Findrisc era $<15$ se le proporcionó educación sanitaria basada en hábitos de vida saludable (anexo I) y factores de riesgo de desarrollar la enfermedad (anexo II) y se le recomendó la repetición del test al cabo de 3 años. Si el paciente lo solicitaba también se le hacia la medición capilar, pero no cuenta para los resultados.

3. Si la puntuación del cuestionario resultaba $\geq 15$, se le propuso realizar una determinación capilar de $\mathrm{HbA} 1 \mathrm{c} 0$ glucemia (basal si el sujeto acudía en ayuno de 8 horas, 0 al azar en caso contrario; si tras realizar la glucemia al azar se obtenía un valor entre 100 y $200 \mathrm{mg} / \mathrm{dl}$ se le realizaba una glucemia basal otro día posterior). Los participantes con glucemia basal mayor o igual a $100 \mathrm{mg} / \mathrm{dL}$ o glucemia al azar mayor o igual a 200 o HbA1c mayor o igual a 5,7\% debían derivarse al médico para su valoración. En la figura 1 se muestra un esquema del procedimiento seguido.

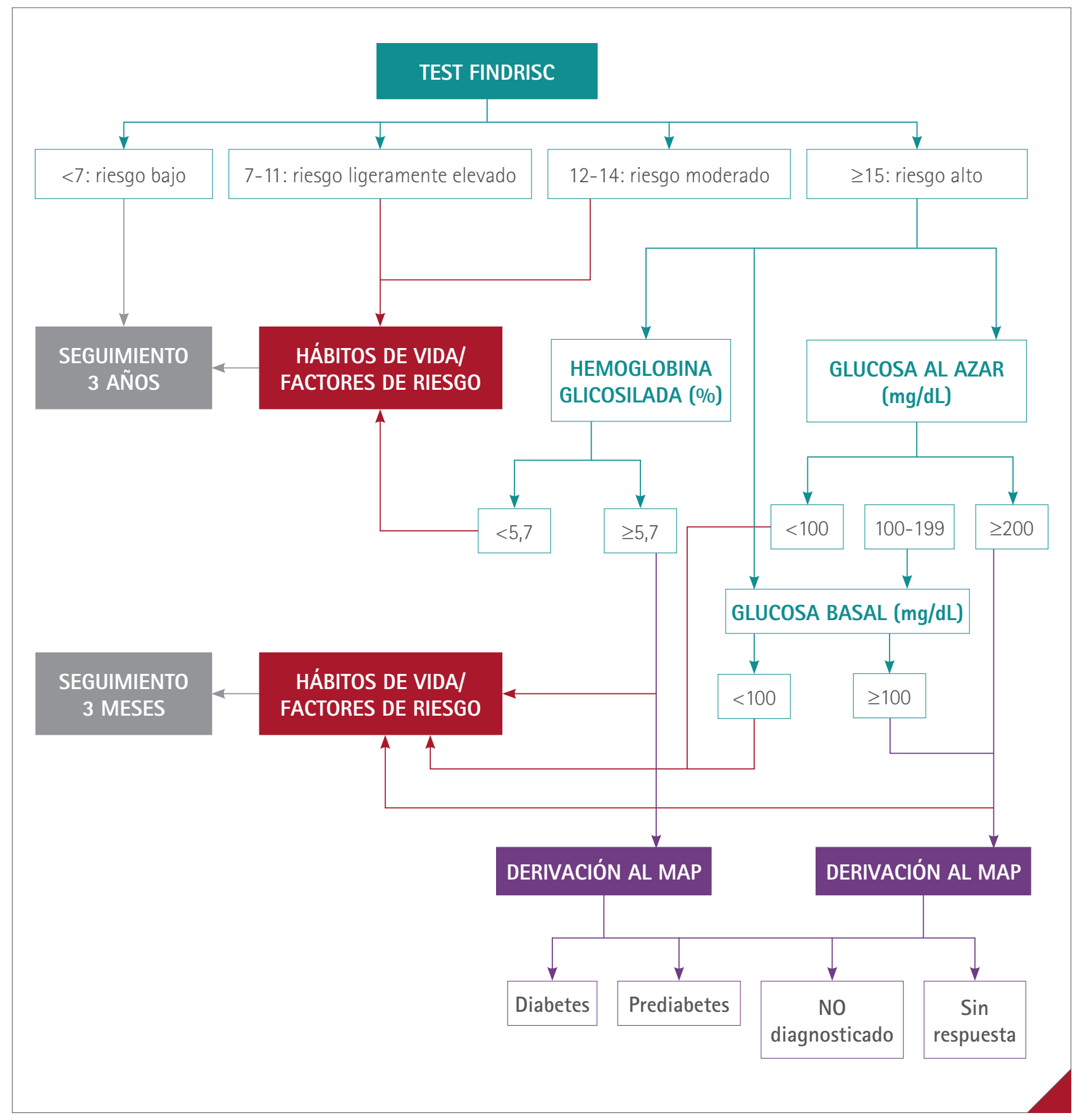

Figura 1 Esquema del procedimiento 


\section{Tratamiento estadístico}

Se calculó la media y desviación estándar (SD) de la puntuación total del test de Findrisc y las frecuencias relativas para cada una de las demás variables categóricas del test y del resultado de las determinaciones capilares, para el conjunto de la muestra, por sexo y edad.

Las diferencias entre grupos se calcularon mediante el test de t de Student, chi cuadrado o Wilcoxon. Los valores referidos de $p$ se basan en test de dos colas con límite de significación estadística de 0,05.

Se solicitó consentimiento informado a todos los participantes. El análisis de los datos para el estudio se realizó de manera anónima y agregada, sin que fuera posible la identificación de los participantes.

\section{RESULTADOS}

En el estudio piloto del programa "Detecta Sucre" del MICOF participaron 31 farmacéuticos de 25 farmacias en la provincia de Valencia.

La media de registros por farmacia fue de 30,1 (SD 19,2). En la figura 2 se muestra un resumen de los resultados.

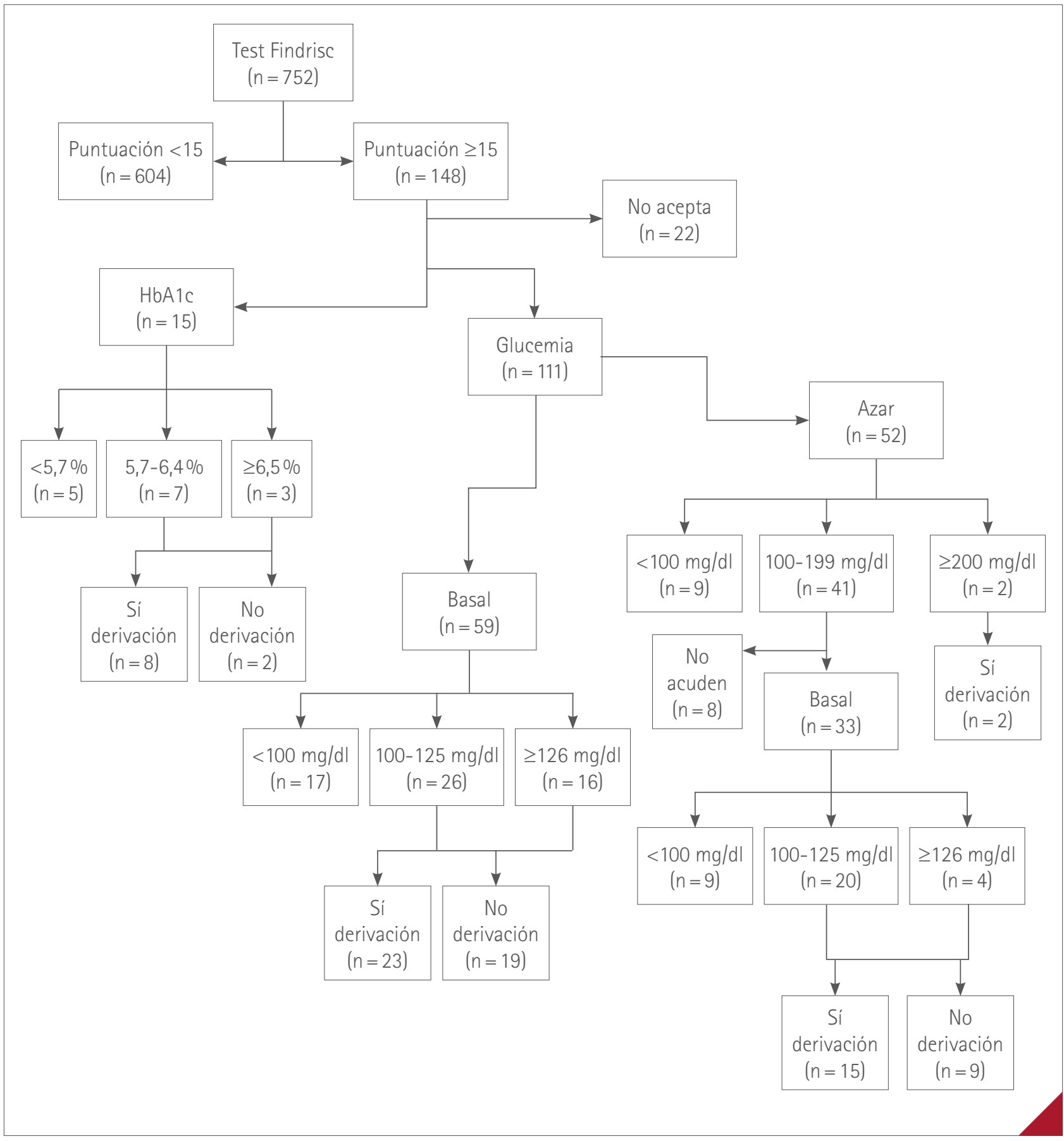

Figura 2 Diagrama de resultados 
Tabla 1 Caracteristicas de la muestra y resultados del test de Findrisc

\begin{tabular}{|c|c|c|c|c|}
\hline Variable (puntos) & $\begin{array}{c}\text { Mujeres n (\% grupo) } \\
\text { (\% total) }\end{array}$ & $\begin{array}{c}\text { Hombres n ( } \% \text { grupo) } \\
\text { (\% total) }\end{array}$ & $\begin{array}{l}\text { Total } n \\
(\% \text { total) }\end{array}$ & $p$ \\
\hline \multicolumn{4}{|l|}{ Edad } & 0,072 \\
\hline $\begin{array}{l}<45(0) \\
45-54(2) \\
55-64(3) \\
>64(4) \\
\text { Total }\end{array}$ & $\begin{array}{c}124(25,8)(16,5) \\
112(23,3)(14,9) \\
117(24,4)(15,6) \\
127(26,5)(16,9) \\
480(100,0)(63,8)\end{array}$ & $\begin{array}{c}76(27,9)(10,1) \\
70(25,7)(9,3) \\
44(16,2)(5,9) \\
82(30,1)(10,9) \\
272(100,0)(36,2)\end{array}$ & $\begin{array}{c}200(26,6) \\
182(24,2) \\
161(21,4) \\
209(27,8) \\
752(100,0)\end{array}$ & \\
\hline \multicolumn{4}{|l|}{ Antecedentes familiares } & 0,452 \\
\hline $\begin{array}{l}\text { No (0) } \\
2^{\circ} \text { Grado (3) } \\
1^{\circ} \text { Grado (5) }\end{array}$ & $\begin{array}{c}231(48,1)(30,7) \\
94(19,6)(12,5) \\
155(32,3)(20,6) \\
480(100,0)(63,8)\end{array}$ & $\begin{array}{c}141(51,8)(18,8) \\
55(20,2)(7,3) \\
76(27,9)(10,1) \\
272(100,0)(36,2)\end{array}$ & $\begin{array}{c}372(49,5) \\
149(19,8) \\
231(30,7) \\
752(100,0)\end{array}$ & \\
\hline \multicolumn{4}{|l|}{ Perímetro de cintura } & 0,002 \\
\hline $\begin{array}{l}M:<80 \mathrm{H}:<94(0) \\
M: 80-88 \mathrm{H}: 94-102(3) \\
M:>88 \mathrm{H}:>102(4)\end{array}$ & $\begin{array}{c}102(21,3)(13,6) \\
136(28,3)(18,1) \\
242(50,4)(32,2) \\
480(100,0)(63,8)\end{array}$ & $\begin{array}{c}85(31,3)(11,3) \\
90(33,1)(12,0) \\
97(35,7)(12,9) \\
272(100,0)(36,2)\end{array}$ & $\begin{array}{c}187(24,9) \\
226(30,1) \\
339(45,1) \\
752(100,0)\end{array}$ & \\
\hline \multicolumn{4}{|l|}{ Actividad física } & 0,002 \\
\hline $\begin{array}{l}\text { Si }(0) \\
\text { No }(2)\end{array}$ & $\begin{array}{c}265(55,2)(35,2) \\
215(44,8)(28,6) \\
480(100,0)(63,8)\end{array}$ & $\begin{array}{c}181(66,5)(24,1) \\
91(33,5)(12,1) \\
272(100,0)(36,2)\end{array}$ & $\begin{array}{c}446(59,3) \\
306(40,7) \\
752(100,0)\end{array}$ & \\
\hline \multicolumn{4}{|c|}{ Consumo fruta, verduras o pan } & 0,007 \\
\hline $\begin{array}{l}\text { Diario }(0) \\
\text { No diariamente (1) }\end{array}$ & $\begin{array}{c}417(86,9)(55,5) \\
63(13,1)(8,4) \\
480(100,0)(63,8)\end{array}$ & $\begin{array}{c}216(79,4)(28,7) \\
56(20,6)(7,4) \\
272(100,0)(36,2)\end{array}$ & $\begin{array}{c}633(84,2) \\
119(15,8) \\
752(100,0)\end{array}$ & \\
\hline \multicolumn{4}{|l|}{ Medicamentos HTA } & 0,003 \\
\hline $\begin{array}{l}\text { Si }(0) \\
\text { No }(2)\end{array}$ & $\begin{array}{c}146(30,4)(19,4) \\
334(69,6)(44,4) \\
480(100,0)(63,8)\end{array}$ & $\begin{array}{c}111(40,8)(14,8) \\
161(59,2)(21,4) \\
272(100,0)(36,2)\end{array}$ & $\begin{array}{c}257(34,2) \\
495(65,8) \\
752(100,0)\end{array}$ & \\
\hline \multicolumn{4}{|l|}{ Glucemia alta previa } & 0,320 \\
\hline $\begin{array}{l}\text { Si (0) } \\
\text { No (5) }\end{array}$ & $\begin{array}{c}60(12,5)(8,0) \\
420(87,5)(55,9) \\
480(100,0)(63,8)\end{array}$ & $\begin{array}{c}41(15,1)(5,5) \\
231(84,9)(30,7) \\
272(100,0)(36,2)\end{array}$ & $\begin{array}{c}101(13,4) \\
651(86,6) \\
752(100,0)\end{array}$ & \\
\hline \multicolumn{4}{|l|}{ IMC } & $<0,001$ \\
\hline $\begin{array}{l}<25(0) \\
25-30(1) \\
>30(3) \\
\text { Total }\end{array}$ & $\begin{array}{c}214(44,6)(28,5) \\
172(35,8)(22,9) \\
94(19,6)(12,5) \\
480(100,0)(63,8)\end{array}$ & $\begin{array}{c}79(29,0)(10,5) \\
129(47,4)(17,2) \\
64(23,5)(8,5) \\
272(100,0)(36,2)\end{array}$ & $\begin{array}{c}293(39,0) \\
301(40,0) \\
158(21,0) \\
752(100,0)\end{array}$ & \\
\hline
\end{tabular}

H: hombre; HTA: hipertensión arterial; IMC: índice de masa corporal; M: mujer.

Se incluyeron en el estudio 752 usuarios, con media de edad de 54,6 (SD 15,0) años. De éstos, 480 (63,8\%) correspondieron a mujeres. Sus características con los resultados de las preguntas del test de Findrisc se presentan en la tabla 1.
El número de usuarios con riesgo alto o muy alto $(F \geq 15)$ fue de 148 (19,7\%) de los 752 encuestados (tabla 2).

El 1,5\% de los usuarios de menos de 45 años tienen riesgo alto y ninguno riesgo muy alto, frente a los usuarios 
de más de 45 años que tienen riesgo alto, el 22,8\%, y riesgo muy alto, el 3,4\% (tabla 3).

Se propuso realizar la medición capilar a 148 pacientes, de los cuales 22 no aceptaron. De los 126 restantes a 15 se les hizo HbA1c, a 59 glucemia basal y 52 glucemia al azar. A 41 pacientes con glucemia al azar se les propuso realizar una glucemia basal al día siguiente, de los cuales 8 no acudieron. Por tanto, el número de mediciones realizadas fue 118. De estas 118 mediciones en 78 el valor obtenido fue superior al considerado normal. De ellos, 48 fueron derivados al médico y 30 no lo fueron.
Si calculamos los porcentajes referidos al total de la muestra 148 pacientes $(19,7 \%)$ dieron riesgo alto o muy alto en el test de Findrisc y $78(10,4 \%)$ tuvieron valores de glucemia o $\mathrm{HbA} 1 \mathrm{c}$ superiores a los considerados normales. El detalle de los resultados puede verse en la figura 2.

Las intervenciones realizadas de acuerdo con el procedimiento descrito se muestran en la tabla 3. En cuanto a las derivaciones al médico, no hubo usuarios de menos de 45 años derivados, mientras que se derivó al $9 \%$ de los mayores de 45 años.

Tabla 2 Riesgo distribuido por sexo

\begin{tabular}{|c|c|c|c|c|}
\hline & $\begin{array}{c}\text { Mujeres } \\
\text { n (\% grupo) (\% total) }\end{array}$ & $\begin{array}{c}\text { Hombres } \\
\mathrm{n}(\% \text { grupo) }(\% \text { total })\end{array}$ & $\begin{array}{c}\text { Total } \\
\text { n (\% total) }\end{array}$ & $\mathrm{p}$ \\
\hline Findrisc [n (SD)] & $10,5(4,8)$ & $10,3(5,0)$ & $10,4(4,9)$ & 0,520 \\
\hline Muy alto > 20 & $12(2,5)(1,6)$ & $7(2,6)(0,9)$ & $19(2,5)$ & \multirow{6}{*}{0,829} \\
\hline Alto $15-20$ & $84(17,5)(11,2)$ & $45(16,5)(6,0)$ & $129(17,2)$ & \\
\hline Moderado 12-14 & $93(19,4)(12,4)$ & $59(21,7)(7,8)$ & $152(20,2)$ & \\
\hline Ligero 7-11 & $190(39,6)(25,3)$ & $98(36,0)(13,0)$ & $288(38,3)$ & \\
\hline Bajo $<7$ & $101(21,0)(13,4)$ & $63(23,2)(8,4)$ & $164(21,8)$ & \\
\hline Total & $480(100,0)(63,8)$ & $272(100,0)(36,2)$ & $752(100,0)$ & \\
\hline
\end{tabular}

SD: desviación estándar.

Tabla 3 Riesgo e intervención en función de la edad

\begin{tabular}{|c|c|c|c|c|}
\hline \multirow[b]{2}{*}{ Variable } & \multicolumn{3}{|l|}{ Intervalos de edad } & \multirow[b]{2}{*}{$p$} \\
\hline & $\begin{array}{c}18-44 \text { años } \\
\text { n (\% grupo) (\% total) }\end{array}$ & $\begin{array}{c}\geq 45 \text { años } \\
\mathrm{n}(\% \text { grupo) (\% total) }\end{array}$ & $\begin{array}{c}\text { Total } \\
\text { n (\% total) }\end{array}$ & \\
\hline Findrisc [m (SD)] & $6,8(3,7)$ & $11,8(4,6)$ & $10,4(4,9)$ & \multirow{7}{*}{$<0,001$} \\
\hline Muy alto > 20 & 0 & $19(3,4)(2,5)$ & $19(2,5)$ & \\
\hline Alto $15-20$ & $3(1,5)(0,4)$ & $126(22,8)(16,8)$ & $129(17,2)$ & \\
\hline Moderado 12-14 & $22(11,0)(2,9)$ & $130(23,6)(17,3)$ & $152(20,2)$ & \\
\hline Ligero 7-11 & $75(37,5)(10)$ & $213(38,6)(28,3)$ & $288(38,3)$ & \\
\hline Bajo $<7$ & $100(50,0)(13,3)$ & $64(11,6)(8,5)$ & $164(21,8)$ & \\
\hline Total & $200(100,0)(26,6)$ & $552(100,0)(73,4)$ & $752(100,0)$ & \\
\hline \multicolumn{5}{|l|}{ Intervención } \\
\hline ES y $F$ a los 3 años & $197(98,5)(26,2)$ & $412(74,6)(54,8)$ & $609(81,0)$ & \\
\hline ES y $\mathrm{F}$ a los 3 meses & $3(1,5)(0,4)$ & $92(16,7)(12,2)$ & $95(12,6)$ & \\
\hline ES, F y derivación al médico & $0(0,0)(0,0)$ & $48(8,7)(6,4)$ & $48(6,4)$ & \\
\hline Total & $200(100,0)(26,6)$ & $552(100,0)(73,4)$ & $752(100,0)$ & \\
\hline
\end{tabular}

SD: desviación estándar; ES: educación sanitaria; F: repetir el test de Findrisc. 
De los 48 (6,4\% del total) pacientes derivados por el farmacéutico, $32(66,7 \%)$ no fueron al médico o el farmacéutico no obtuvo respuesta tras la derivación. De los 16 cuyo resultado se conoce, en $3(18,8 \%)$ el médico no tuvo en cuenta nuestro informe y no midió la glucemia, 1 (6,2\%) obtuvo un valor dentro de la normalidad, 7 (43,8\%) fueron diagnosticados de prediabetes y $5(31,2 \%)$ lo fueron de DM2. Extrapolando los datos a la muestra total, en el caso de que se dispusiera de la información sobre todos los derivados al médico, los diagnósticos de DM supondrían un $2,0 \%$ y los de prediabéticos el 2,8\%.

De los 95 pacientes cuya intervención fue educación sanitaria y repetición del test a los 3 meses, 9 (9,5\%) regresaron a la farmacia. La media en la reducción de las variables perímetro de cintura, IMC, peso y puntuación Findrisc a los 3 meses se presentan en la tabla 4.

Se realizaron 207 mediciones no incluidas en el protocolo a petición de los pacientes. De todas ellas 45 (21,7\%) obtuvieron valores superiores a los considerados normales, a pesar de tener riesgo moderado o menos en el test de Findrisc.

\section{DISCUSIÓN}

Los resultados de este estudio piloto dejan entrever la utilidad de la farmacia comunitaria, y especialmente la del farmacéutico comunitario, como agente sanitario con un papel importante en el cribado de DM2 al detectar, en el presente trabajo, casi un $20 \%$ de usuarios con un riesgo elevado de padecer la enfermedad.

La cifra de diagnósticos de nuevos pacientes diabéticos y prediabéticos obtenida a través del servicio, estimada en el $31,2 \%$ y $43,8 \%$ de las derivaciones respectivamente (equivalente al $2,0 \%$ y $2,8 \%$, respectivamente del total de la muestra), y la reducción de la medida de las variables de perímetro de cintura, IMC, peso y puntuación Findrisc a los 3 meses tras intervención del farmacéutico, demuestran la utilidad del farmacéutico comunitario tanto en el cribado de DM2 y preDM2 como en la educación sanitaria de estas personas. Derivar a estas personas al médico, del que podrán recibir en su caso un diagnóstico, o bien información, educación sanitaria, seguimiento y control, consideramos que puede contribuir a retrasar la aparición o progreso de la enfermedad y la manifestación de complicaciones.

Las características de la muestra son similares a las encontradas en otros estudios llevados a cabo en farmacias comunitarias (12-16,19). Más del $63 \%$ de los participantes son mujeres, más del $73 \%$ del total de encuestados tienen más de 45 años y también más del $75 \%$ tienen valores de perimetro de cintura superiores a la normalidad y más del $60 \%$ presentan sobrepeso u obesidad, que es mayor entre las mujeres. Estas cifras son similares a las estimadas para
Tabla 4 Reducción de la medida en las variables a los 3 meses

\begin{tabular}{|l|c|c|}
\hline Variable [m (SD)] & PRE-POST & $p$ \\
\hline Perimetro de cintura & $2,7(2,9)$ & 0,024 \\
\hline IMC & $1,2(1,7)$ & 0,063 \\
\hline Peso & $3,1(4,6)$ & 0,079 \\
\hline Puntuación Findrisc & $0,9(2,7)$ & 0,347 \\
\hline
\end{tabular}

SD: desviación estándar; IMC: índice de masa corporal.

la población adulta en España (18), donde la prevalencia de sobrepeso y obesidad fue del 39,3 y 21,6\%, respectivamente.

Más del 59\% afirma realizar al menos 30 minutos diarios de ejercicio y más del $84 \%$ afirma consumir verduras 0 fruta todos los días. Es muy posible, no obstante, que estas respuestas estén magnificadas por la autopercepción del paciente, por lo que la puntuación obtenida en el test podría ser ligeramente mayor que el obtenido y, por lo tanto, realmente habría un número mayor que el detectado de sujetos con riesgo alto o muy alto.

Las cifras de riesgo alto y muy alto de desarrollar DM2 encontradas en nuestro estudio $(19,7 \%)$ son similares a otros estudios en farmacias comunitarias utilizando el mismo punto de corte $F \geq 15(13,14,16)$; de ellos el $98 \%$ corresponde a sujetos mayores de 45 años.

Aunque en el procedimiento se estableció que la determinación de la glucemia capilar se haría solamente a los participantes con resultado del cuestionario $F \geq 15$ (148), la presión de los usuarios obligó a los farmacéuticos a realizarla a un número mayor de participantes (367).

En un número un tanto elevado,14 (el 42,4\%) de las $\mathrm{HbA} 1 \mathrm{c}$ realizadas, se encontraron cifras entre 5,7 y $6,4 \%$, y 106 personas (el 35,5\%) de las glucemias realizadas presentaron cifras entre 100 y $125 \mathrm{mg} / \mathrm{dL}$. Son cifras más elevadas que las encontradas en el estudio di@bet.es (14,8\%) (2). Se trataría de un estado de prediabetes, que se relaciona a mayor riesgo de desarrollar DM y de padecer complicaciones cardiovasculares en el futuro (10), pero cuyo conocimiento concede al paciente la posibilidad de instaurar medidas de cambio de hábitos que eviten o retrasen la evolución de la DM2.

Las intervenciones con los pacientes no derivados al médico ( $F<15$ y $F \geq 15$ con $\mathrm{HbA} 1 \mathrm{c}<5,7 \%$ o $\mathrm{GBC}<100 \mathrm{mg} / \mathrm{dL}$ ) se encaminaron a conseguir que los participantes en el cribado tomaran conciencia de su situación con el fin de iniciar las actuaciones preventivas y de control de su evolución.

Una de las limitaciones del presente estudio estriba, precisamente, en no haber tenido un retorno de todos los pacientes derivados al médico, bien por la idiosincrasia del paciente, bien por fallos en la comunicación, o bien por no estar los médicos informados sobre el programa. Los cribados de diabéticos ocultos en farmacia 
comunitaria en España $(12-14,16)$ no siempre han tenido información del diagnóstico por la falta de retorno del médico; en nuestro estudio solo el $37,8 \%$ retornaron. Este hecho debe ser tenido en cuenta para futuros trabajos de esta índole.

Otra limitación de nuestro estudio es que los resultados son válidos tan solo para el conjunto de usuarios de la farmacia, ya que la muestra no es representativa de la población nacional, lo que no era objetivo del estudio al tratarse de un programa piloto. Por tanto, los resultados no se pueden extrapolar a la población general y deben interpretarse con precaución a la espera de contar con una muestra más elevada y un estudio que supere las limitaciones del presente. Además, el presente trabajo proviene de la voluntariedad de la participación tanto de los farmacéuticos como de los usuarios. Esto puede que haya alterado los resultados en tanto los farmacéuticos pudieron presentar una tendencia a reclutar a aquellos pacientes con un perfil susceptible de presentar riesgo alto. No obstante, para el objetivo principal del estudio, valorar la viabilidad de un servicio de cribado de diabetes en la farmacia comunitaria, la muestra es perfectamente válida ya que sí es representativa de la que acude normalmente a las farmacias comunitarias.

Por su parte, las mejoras en parámetros tras la intervención farmacéutica pudieron mejorar en estas personas por el hecho de estar siendo seguidas por un profesional sanitario. Requiriéndose, para afirmar con mayor rotundidad que la intervención fue plenamente eficaz, un seguimiento más largo en el tiempo.

La cifra de nuevos diagnósticos de pacientes diabéticos obtenida en nuestro estudio, estimada en el 30,7\% de las derivaciones, equivalente al 2,8\% del total de la muestra, demuestra la eficiencia de la farmacia y el farmacéutico comunitario en un cribado de diabéticos ocultos como el que aquí se presenta.

Aun teniendo en cuenta que se trata de un estudio piloto, la implantación del cribado de DM2 en la farmacia comunitaria como un servicio profesional farmacéutico asistencial (21) retribuido permitiría un importante ahorro económico al sistema sanitario público al anticipar su atención a estadios más tardíos de la enfermedad, cuando el abordaje terapéutico ha de ser más agresivo. Además, el tratamiento temprano puede propiciar la integración de los nuevos diabéticos en programas multidisciplinares de atención integral a estos pacientes con el consecuente retraso de la aparición de complicaciones macro y microvasculares.

\section{AGRADECIMIENTOS}

A los 31 farmacéuticos comunitarios de las 25 farmacias que participaron en este estudio.

\section{REFERENCIAS BIBLIOGRÁFICAS}

1. Encuesta Nacional de Salud España 2017. [Internet] [consultado 13 Dic 2018]. Disponible en: https://www.mscbs.gob.es/estadEstudios/ estadisticas/encuestaNacional/encuestaNac2017/ENSE17_pres_ web.pdf

2. Soriguer F, Goday A, Bosch-Comas A, Bordiú E, Calle-Pascual A, Carmena $R$, et al. Prevalence of diabetes mellitus and impaired glucose regulation in Spain:The Di@bet.es Study. Diabetologia. 2012;55:8893. doi:10.1007/s00125-011-2336-9

3. Generalitat Valenciana. Conselleria de Sanitat Estudio Valencia: investigación de la prevalencia de diabetes mellitus y sindrome metabólico, 2010. Disponible en: http://publicaciones.san.gva.es/publicaciones/documentos/V.2672-2010.pdf

4. Fornos Pérez JA, Fernández Cordeiro M, Garcia Rodríguez P, González Añón D, Andrés Iglesias J, Andrés Rodríguez NF. Análisis de la utilización de medicamentos por los pacientes diabéticos en la provincia de Pontevedra. Av Diabetol. 2010;26:47-52. doi:10.1016/ S1134-3230(10)61010-1

5. Lindenmeyer $A$, Hearnshaw $H$, Vermeire $E$, van Royen $P$, WensJ, Biot $Y$. Interventions to improve adherence to medication in people with type 2 diabetes mellitus: A review of the literatura on the role of pharmacists. J Clin Pharm Ther. 2006;31:409-19. doi:10.1111/j.13652710.2006.00759.x

6. American Diabetes Association. Diagnosis and classification of diabetes mellitus. Diabetes Care 2014; 37 (Suppl. 1):S81-S90. doi:10.2337/dc14-S081

7. Soriguer F, Valdés S, Tapia MJ, Esteva I, de Adana MS, Almaraz MC, Morcillo S, Fuentes EG, Rodríguez F, Rojo-Martinez G. Validación del FINDRISC (FINnish Diabetes Risk SCore) para la predicción del riesgo de diabetes tipo 2 en una población del sur de España. Estudio Pizarra. Medicina Clínica. 2012 Apr 14;138(9):371-6. doi:10.1016/j. medcli.2011.05.025

8. National Institute for Health and Care Excellence (NICE) [Internet]. Preventing type 2 diabetes: Risk identification and interventions for individuals at high risk. 2012 [consultado 13 Dic 2018]. Disponible en: http://www.nice.org.uk/guidance/ph38

9. Pottie K, Jaramillo A, Lewin G, Dickinson J, Bell N, Brauer P, et al., Canadian Task Force on Preventive Health Care. Recommendations on screening for type 2 diabetes in adults. CMAJ. 2012 Oct 16;184(15):1687-96. doi:10.1503/cmaj.120732

10. Mata M, Artola S, Escalada J, Ezkurra P, Ferrer JC, Fornos JA, et al. Consenso sobre la detección y el manejo de la prediabetes. Grupo de trabajo de consensos y guías clínicas de la Sociedad Española de Diabetes. Av Diabetol. 2015;31:89-101. doi:10.1016/j.avdiab.2014.10.007

11. Fundación para la diabetes. La diabetes se puede prevenir. Informe de resultados. Diciembre 2015. [consultado 14 Dic 2018]. Disponible en: https://www.fundaciondiabetes.org/general/actividad/43/ informe-de-resultados-de-la-campana-la-diabetes-se-puede-prevenir-segun-el-test-findrisk

12. Fornos Pérez JA, Andrés Rodríguez NF, Andrés Iglesias JC, Acuña Ferradanes A, Costas González D, Mera Gallego R. Detección de pacientes con riesgo de desarrollar diabetes en farmacias comunitarias de Pontevedra. Farmacéuticos Comunitarios. 2013;5:141-6. Disponible en: https://www.farmaceuticoscomunitarios.org/es/system/files/ journals/574/articles/5-4-141-146.pdf

13. Pérez JA, Andrés-Rodríguez NF, Lorenzo-Veiga B, Huarte-Royo J, Vivar-Fernández M, Andrés-Iglesias JC, García-Rodríguez P. Detección de personas en riesgo de padecer diabetes en farmacias comunitarias españolas. Farmacéuticos comunitarios. 2015 Jun 30;7(2):1424. doi:10.5672/FC.2173-9218.(2015/Vol7).002.03

14. Fornos-Pérez JA, Andrés-Rodríguez NF, Andrés-Iglesias JC, Luna-Cano R, García-Soidán J, Lorenzo-Veiga B, Mera-Gallego R, García-Riestra 
R. Detección de personas en riesgo de padecer diabetes en farmacias comunitarias de Pontevedra (DEDIPO). Endocrinología y Nutrición. 2016 Oct 1;63(8):387-96. doi:10.1016/j.endonu.2016.06.002

15. Fikri-Benbrahim $N$, Martínez-Martínez $F$, Saéz-Benito $L$, Luque BS, Corpas JP, Moullin JC, Sabater-Hernández D. Assessment of a screening protocol for type 2 diabetes in community pharmacy. The DiabNow Study. Diabetes research and clinical practice. 2015 Jun 1;108(3):e49-52. doi:10.1016/j.diabres.2015.03.006

16. Ruiz D, Climent MT, Salar L, Baixauli VJ, Vicente, Colomer V, Moreno L, Vicedo C, Alcázar S, Lorenzo A, Jiménez J. Diprova 1. Detección e intervención en grupo de riesgo de padecer diabetes en la provincia de Valencia. Farmacéuticos Comunitarios. 2016 May 26; 8 (Suplemento 1). Disponible en: https://www.farmaceuticoscomunitarios. org/sites/default/files/suplemento/pdf/105.pdf

17. Garcia O, Colomer V, Ruiz ED, Pérez C, Baixauli VJ, Climent MT, Salar L, Gil M, Gómez C. Diseño y desarrollo del servicio de cribado de diabetes y prediabetes del MICOF de valencia. Farmacéuticos Comunitarios. 10 (Suplemento 1): 218. Disponible en: https://www.farmaceuticoscomunitarios.org/sites/default/files/suplemento_18/pdf/261.pdf
18. Aranceta-Bartrina J, Pérez-Rodrigo C, Alberdi-Aresti G, Ramos-Carrera N, Lázaro-Masedo S. Prevalencia de obesidad general y obesidad abdominal en la población adulta española (25-64 años) 20142015: estudio ENPE. Rev Esp Cardiol. 2016; 69:579-87. doi:10.1016/j. recesp.2016.02.010

19. Guerra Garcia MM, Fornos Pérez JA, Andrés Rodríguez NF. Detección de diabéticos no diagnosticados en las farmacias comunitarias de la provincia de Pontevedra. Pharm Care Esp. 2003;5(4):166-169.

20. Arias $J L$, Linares FM, Santamaria JM. Detección de posibles diabéticos no diagnosticados en una farmacia comunitaria. Ars Pharmaceutica (internet). 2007;48(2):187-200. Disponible en: https://revistaseug.ugr.es/index.php/ars/article/ view/4985/4792

21. Foro de Atención Farmacéutica-Farmacia Comunitaria (Foro AF-FC). Guía práctica para los Servicios Profesionales Farmacéuticos Asistenciales en la Farmacia Comunitaria. Madrid: Consejo General de Colegios Oficiales de Farmacéuticos; 2019. Disponible en: https:// www.sefac.org/system/files/2021-02/AF_GUIA_SPFA_FORO_2021_ ONLINE_PGs.pdf 
Anexo I. Hábitos de vida saludable

\section{Ejercicio}

La actividad física debe ser adaptada a la edad, capacidad, gustos y posibilidades de cada persona.

Se recomienda un mínimo de 30 minutos al día de ejercicio aeróbico moderado (caminar, ir en bici, subir escaleras, etc.) al menos 5 días a la semana.

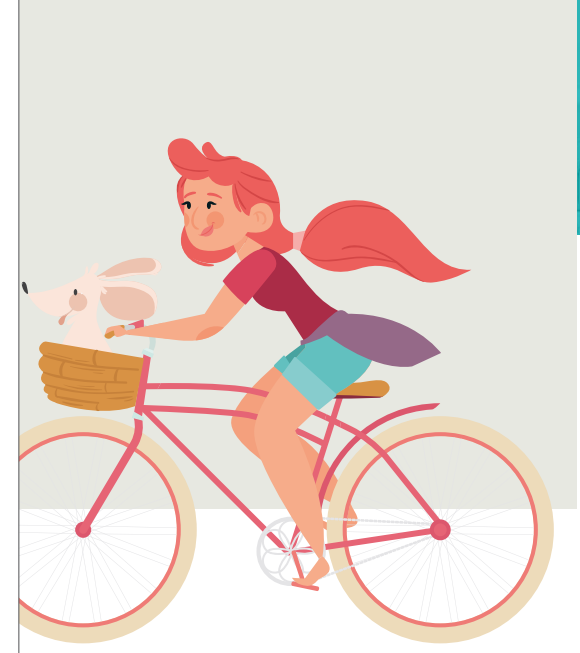

Tu farmacéutico a tu lado \#consejosmicof
Hábitos de vida saludables

Alimentación y ejercicio

\section{MICOF}

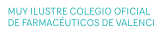

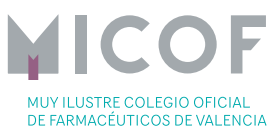

INFORMACIÓN DEL CURSO

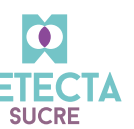

\section{Alimentación}

La variedad, los productos frescos y

locales y la moderación en la cantidad (tamaño de raciones) son principios básicos que favorecen una alimentación saludable.

Las técnicas culinarias empleadas en la preparación de los alimentos pueden afectar a su calidad nutricional. Como norma general, el cocinado al vapor, horno, las preparaciones seguras en crudo o las menos elaboradas ofertan un mejor valor nutricional.
Las líneas básicas en una alimentación equilibrada pueden resumirse en lo que se entiende por "dieta mediterránea". Algunos de los aspectos más relevantes son:

Fraccionar la ingesta a 3-5 comidas al día, evitando ingestas copiosas.

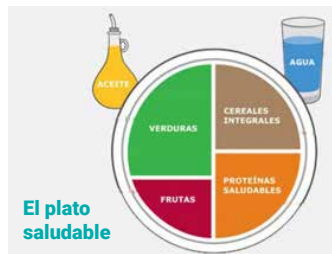

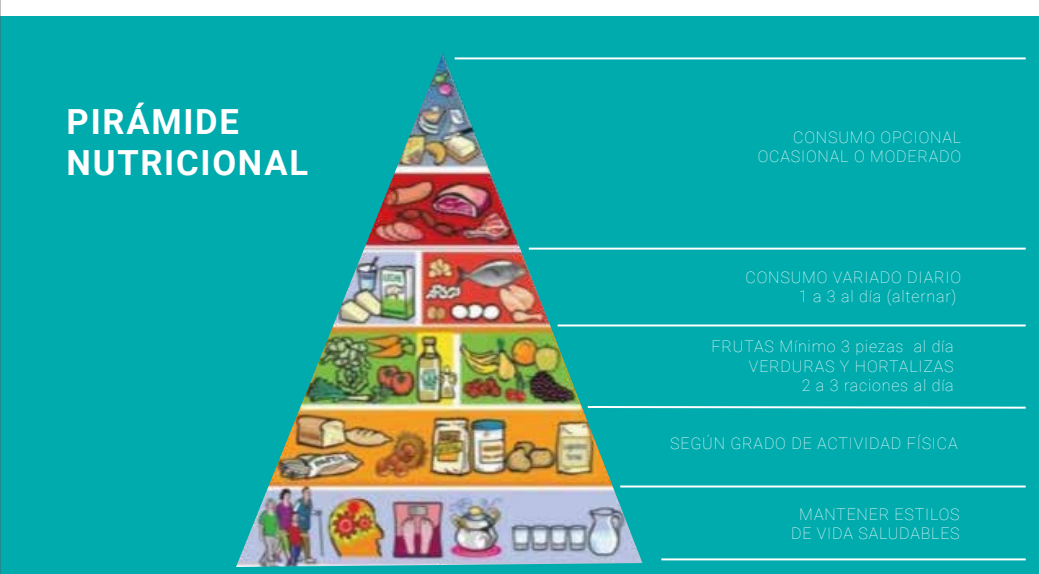

Evitar los azúcares de absorción rápida (azúcares refinados y repostería en general).

- Utilizar alimentos con hidratos de carbono de absorción lenta (legumbres, pasta pan, patatas, arroz, etc.).

- Utilizar alimentos ricos en fibra como las verduras y hortalizas. Se recomienda incorporar al menos 2-3 raciones cada día.

- Consumir un mínimo de 3 piezas de fruta al día, preferiblemente entera.

Es importante la ingesta diaria de 6-8 vasos de agua u otros líquidos (infusiones, zumos, caldos y sopas).

- Limitar los alimentos de origen animal, procurando además que el consumo de pescado (2-3 raciones/semana y al menos una que sea pescado azul) supere al de carne (1-2 raciones/semana y que sea carne blanca y no roja).

El consumo de grasas que están contenidas en embutidos, quesos, carnes en general, mantecas y margarinas debe ser de manera ocasional.

- Utilizar aceite de oliva virgen extra, tanto en preparaciones culinarias como para su consumo en crudo. 
Anexo II. Factores de riesgo de desarrollar DM2

\section{Otros factores}

Otros factores no son modificables, es decir, no dependen de nuestra voluntad:

Edad. El riesgo de desarrollar diabetes tipo 2 aumenta a partir de los 45 años.

Historial familiar con diabetes. Antecedentes de diabetes en familiares de primer grado (padres, hermanos, hijos o abuelos) y también de segundo grado (tíos o sobrinos).

Historial con diabetes gestacional. En el caso de haber tenido diabetes gestacional en algún embarazo anterior.

Origen étnico. Los afroamericanos, latinos/hispanos, indígenas americanos, indígenas de Hawái, isleños del Pacífico y estadounidenses de origen asiático tienen un riesgo más alto de tener diabetes tipo 2 .

Mujeres diagnosticadas de síndrome del ovario poliquístico.
Factores de riesgo de desarrollar DM2

\section{Diabetes Mellitus tipo 2}

Tu farmacéutico a tu lado \#consejosmicof

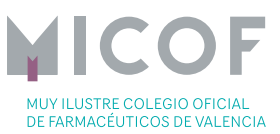

INFORMACIÓN DEL CURSO

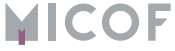

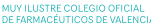

0

\section{Diabetes}

Mellitus tipo 2

\section{DM2}

En cualquier fase de la vida es de gran importancia incidir en una buena educación en salud para mejorar nuestros hábitos de vida desde la infancia y prevenir la aparición de diferentes enfermedades, como la diabetes. Todo ello con el objetivo de aumentar nuestra esperanza de vida y la calidad de la misma.

\section{Factores de riesgo}

Existen muchos factores de riesgo en la diabetes tipo 2 y adquiriendo hábitos de vida saludable podemos disminuir los factores de riesgo modificables, como son:

- Obesidad. Es el factor de riesgo más importante para la diabetes tipo 2 Cuanto mayor sea el sobrepeso, mayor será la resistencia del cuerpo a la insulina

- Falta de ejercicio. Una vida sedentaria no permite mantener un buen estado físico, con las posibles consecuencias que ello conlleva.
- Hipertensión arterial. valores de presión arterial por encima de 140/90 $\mathrm{mmHg}$.

- Alteraciones en el colesterol. HDL menor de $35 \mathrm{mg} / \mathrm{dl}$ y/o triglicéridos por encima de $250 \mathrm{mg} / \mathrm{dl}$.

- Tabaquismo. Fumar aumenta el riesgo de diabetes tipo 2 .

- Dieta poco saludable y equilibrada no teniendo en cuenta un control de la ingesta de hidratos de carbono y grasas saturadas.

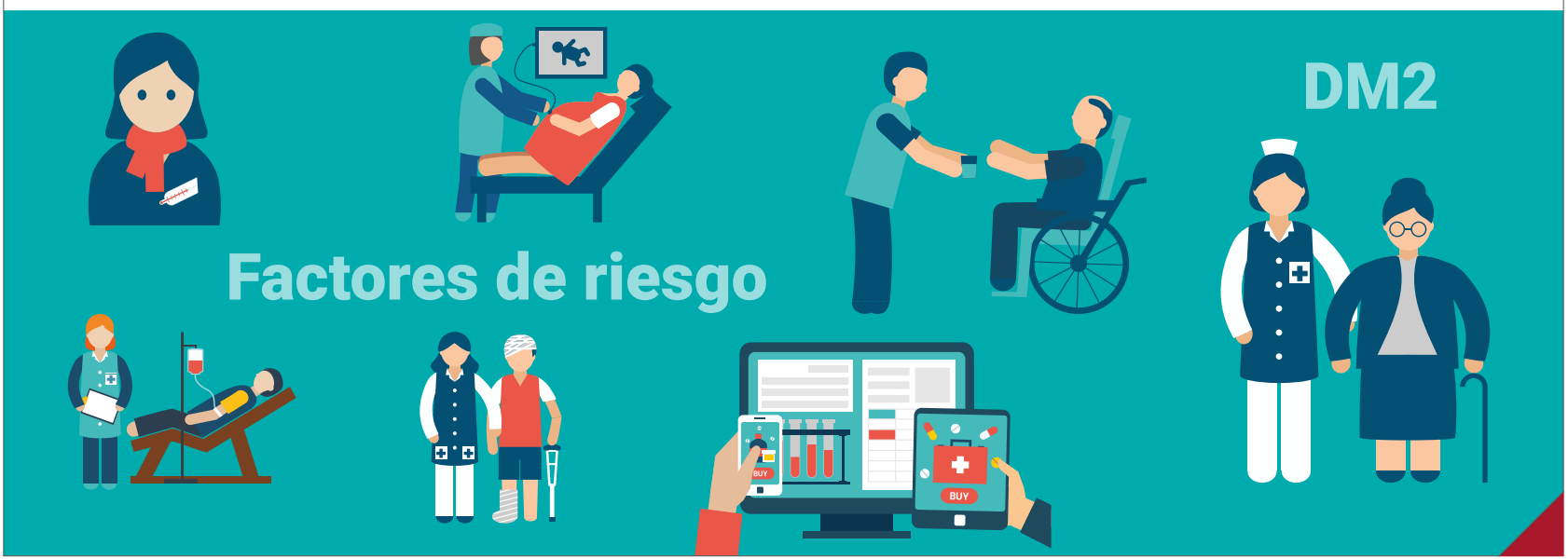

